\title{
Due Novelle Del Decameron
}

\author{
LuCIA STRAPPINI*
}

ABSTRACT: La lettura del Decameron che propongo è concentrata specificamente su due novelle, la prima e la decima della quarta giornata (IV, 1 e IV, 10), che presentano molte ragioni di interesse nella trama, nella caratterizzazione dei personaggi e nei motivi di fondo della narrativa, fondata sulla congiunzione di tragico e di comico in quanto tratto massimamente significante della vicenda umana sotto tutti i profili.

PAROLE CHIAVE: Decameron; parodia; tragico; comico

RESUMO: A leitura aqui proposta do Decameron concentra-se especificamente em duas novelas: a primeira e a décima da quarta jornada (IV, 1 e IV, 10), que apresentam muitos pontos de interesse pela trama, pela caracterização dos personagens e pelos temas centrais da narrativa, fundada na conjunção e complementaridade de trágico e cômico como traços máximos e significantes da aventura humana sob todos os aspectos.

PALAVRAS-CHAVE: Decameron; paródia; trágico; cômico

ABSTRACT: The proposed interpretation of The Decameron is specifically based on two stories, the first and the tenth day of the fourth story (IV 1 and IV, 10), presenting many points of interest to the plot, to create the characters and the central

\footnotetext{
* Università per gli Stranieri di Siena
} 
theme of the narrative, founded on the tragic and comic conjunction, as the most significant traces of the human adventure in all aspects.

KEYWORDS: Decameron; parody; tragic; comic 


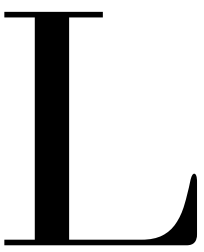

a lettura del Decameron che propongo è concentrata specificamente su due novelle, la prima e la decima della quarta giornata (IV, 1 e IV, 10), che presentano molte ragioni di interesse, come si vedrà, nella trama, nella caratterizzazione dei personaggi e nei motivi di fondo. Ma, ancora prima di addentrarci nell' analisi puntuale, è opportuno considerare che si trovano qui concentrati svariati punti e temi che percorrono l'intero libro. Naturalmente questo aspetto non è riscontrabile solo in questo caso; al contrario, come si può verificare per tutte le grandi opere, i motivi conduttori attivi nel tessuto complessivo dell'opera si riverberano, in maniere diverse, anche su parziali spaccati o frammenti testuali che si prestano ad esami ravvicinati dei temi e dei motivi dell'opera in oggetto meglio e più delle letture critiche di carattere panoramico o trasversale. 
Il nesso che lega in profondo le due novelle che propongo è rappresentato dalla parodia. L'idea, il concetto di parodia sono presenti e attivi nella letteratura classica e poi nella cultura moderna in svariate accezioni e funzionalità. Bisognerebbe perciò ridefinirne il senso e l'uso per verificarne l'esistenza e la concreta, puntuale operatività che, a mio parere, assume un carattere diverso per ogni contesto e in riferimento a ciascuna opera. Mi limito qui a utilizzare la parola e il suo concetto nel senso che preciserò in seguito.

Dunque, si è scritto molto sull'attitudine parodistica del Boccaccio del Decameron, per affermarne la forza attiva o per negarne il rilievo nell'ideazione compositiva e nella strutturazione narrativa. A me sembra che non sia discutibile l'attitudine boccacciana, nel Decameron, a concepire l'intero universo rappresentato (perché di universo si tratta, nella sua visione) sotto il profilo della parodia. A cominciare proprio dall'Introduzione con la descrizione della peste che si dispone precisamente tra citazioni, ricalchi, suggestioni letterarie relative all' argomento e richiami espliciti a una veridicità degli eventi - "A me medesimo incresce andarmi tra tante miserie ravolgendo [...] addivenne, sì come io poi da persona degna di fede sentii"2 (BOCCACCIO, Introduzione1987, p. 49,corsivomio- convalidata dalla diretta testimonianza del narratore - "il che, se dagli occhi di molti e da' miei non fosse stato veduto, appena che io ardissi di crederlo, non che di scriverlo, quantunque da fededegna udito l'avessi" (ibid., p. 16) -, che mirano, per un verso, a rafforzare appunto il carattere realistico dei fatti narrati, ma, insieme, a prospettarli in una dimensione altra che va precisamente al di là di quel significato letterale immediato che l'asserzione di veridicità rivendica. Il senso della citazione, del calco, viene dunque, in questo modo, rovesciato nella direzione opposta. Realismo (nella peculiare accezione decameroniana) versus letterarietà: l'uno opposto all'altra, l'uno in funzione dell'altra.

È una linea doppia, ambigua, sulla quale si dispone la materia narrata che trova un primo e chiaro riscontro proprio nella novella che apre le giornate e la raccolta, quella di ser Ciappelletto, condotta coerentemente sul filo dell'inversione. Insomma io credo che la parodia rappresenti effettivamente una chiave di lettura rilevante per comprendere il senso dell'opera o, almeno, uno dei sensi, visto che sarebbe un'operazione certamente superficiale quella che volesse ridurre a una sola unità il complesso dei suoi intendimenti concettuali, letterari e culturali. Allora, pur tenendo presenti queste avvertenze, si può ben vedere come sia ampiamente operativa nel tessuto del Decameron una tensione parodistica come riscrittura di modelli, fonti, riferimenti della più ampia tradizione letteraria (orale e scritta, italiana ed europea, classica e moderna) e anche come autoparodia, ovvero autocitazione (basterebbe pensare ai nomi dati ai dieci novellatori, allusivi anche a personaggi delle sue opere predecameroniane) o riscrittura di propri testi.

2 Le fonti letterarie, classiche e medievali, sono state puntualmente indicate dalla critica decameroniana. Cfr. l'edizione del Decameron, curata da V. Branca (1987). 
Nella IV giornata del Decameron, come è ben noto, "si ragiona di coloro li cui amori ebbero infelice fine". In piena sintonia con il tema, la novella che apre la giornata $(\mathrm{IV}, 1)$ è modulata su una tonalità tragica che definirei assoluta, ovvero priva di qualunque contrappeso ironico, comico o umoristico; e su questa linea si collocano le successive fino all'ultima che, come sempre, è assegnata a Dioneo, sciolto per decisione comune dal vincolo tematico e le cui narrazioni sono improntate sempre alla maggiore libertà da ogni obbligo sia per il contenuto che per il tono del racconto.

Si registra in questo caso un fenomeno singolare: Dioneo racconta in chiusura di giornata una novella che non solo si allontana dal tema comune, in virtù del suo "privilegio", ma che, in più, si presenta con tutta evidenza come una versione comica della vicenda tragica di Tancredi e Ghismonda racchiusa nella prima, ovvero un rovesciamento, un ribaltamento che non può che essere intenzionale, data la loro collocazione (inizio e fine giornata) e l'opposizione nitida dei caratteri dei due rispettivi novellatori: Fiammetta, per la I, il personaggio (di questa opera e dell'altra, Elegia di Madonna Fiammetta, nella quale Boccaccio aveva analizzato le sottili manifestazioni della passione amorosa) che più degli altri incarna il moto passionale e Dioneo, per l'ultima della giornata, personaggio che fin dall'inizio si propone come "oltre a ogni altro era piacevole e pieno di motti" e per questo sciolto nel novellare, come si diceva, dal vincolo del tema della giornata. Del resto proprio a Dioneo è riservato il compito di chiudere le giornate e l'intera raccolta con la novella di Gualtieri e Griselda $(X, 10)$ nella quale, come ho mostrato in altra occasione (STRAPPINI, 2010), viene ribaltato nelle considerazioni finali comiche e parodistiche, perfino scurrili, il tono dominante della vicenda che è stata raccontata, in costante tensione sul filo della più accentuata drammaticità e melodrammaticità.

Tornando alle due novelle a confronto, possiamo aggiungere altri elementi agli indizi del loro rapporto intenzionale e strutturato, nel segno dell'affinità o del suo contrario. Una prima analogia riguarda il luogo di svolgimento della storia (la città di Salerno /il principato di Salerno), luogo privo naturalmente di connotati storici e geografici determinati e dunque proiezione dell'immaginazione fantastica. Ancora, al principe di Salerno, Tancredi, corrisponde un insigne cittadino come il "grandissimo medico" Mazzeo della Montagna. Entrambi uomini illustri, anziani e socialmente degni, i quali però nella dimensione privata, nella veste di padre, il primo, e di marito, il secondo, incarnano pienamente il loro ruolo di padri-padroni, imponendo alle donne che sono, per legge, a loro sottomesse la propria assoluta volontà. Tancredi, pur avendo a che fare con una "bellissima del corpo e del viso quanto alcuna altra femina fosse mai, e giovane e 
gagliarda e savia più che a donna per avventura non si richiedea" (IV, 1, 5) e Mazzeo, ugualmente legato a una "bella e gentil giovane" (IV, 10, 4), non vogliono o non riescono a soddisfare le esigenze del tutto naturali delle due donne. Ghismonda ribalterà sul padre in una vera e propria arringa le accuse che da lui le sono state rivolte: "Sono adunque, sì come da te generata, di carne, e sì poco vivuta, che ancor son giovane, e per l'una e per l'altra piena di concupiscibile disidero" (IV, 1, 34). Dell'altra, meno consapevole e moralmente determinata, si registra comunque lo scontento: "Di che ella viveva pessimamente contenta" (IV, 10,5). Dunque entrambe le giovani prendono coscienza della violenza oppressiva a cui sono sottoposte all'interno del legame familiare ed entrambe, nel nome delle legittime esigenze naturali, decidono di trovare il modo di soddisfarle; le modalità sono, per molti versi, differenti, in conformità con il loro status e la loro intelligenza individuale e sociale. Ghismonda infatti

dimorando col tenero padre, sì come gran donna, in molte dilicatezze, e veggendo che il padre, per l'amor che egli le portava, poca cura si dava di più maritarla, né a lei onesta cosa pareva il richierdernelo, si pensò di volere avere, se esser potesse, occultamente un valoroso amante. E veggendo molti uomini nella corte del padre usare, gentili e altri, sì come noi veggiamo nelle corti, e considerate le maniere e' costumi di molti, tra gli altri un giovane valletto del padre, il cui nome era Guiscardo, uom di nazione assai umile ma per vertù e per costumi nobile, più che altro le piacque, e di lui tacitamente, spesso vedendolo, fieramente s'accese, ognora più lodando i modi suoi. (IV, 1, 6, corsivo mio).

Mentre la moglie del medico, "savia e di grande animo"

si dispose di gittarsi alla strada e voler logorar dell'altrui; e più e più giovani riguardati, alla fine uno ne le fu all'animo, nel quale ella pose tutta la sua speranza, tutto il suo animo e tutto il ben suo. (IV, 10, 6, corsivo mio).

C'è un evidente nesso tra le due azioni-reazioni e consiste nel carattere razionale, meditato, riflessivo che sostiene le modalità di soddisfacimento del desiderio. E tuttavia proprio nell'accorta ricerca dell' amante adatto si registra un primo segno del carattere distintivo della seconda novella, nel verso del preciso rovesciamento del modello. Ghismonda sceglie infatti 
un giovane della corte del padre, "uom di nazione assai umile ma per vertù e costumi nobile", si preoccupa di non dare scandalo ("occultamente"), di non pregiudicare la dignità pubblica del padre/sovrano. La moglie del medico invece fa la sua scelta disponendo "di gittarsi alla strada" e qui trova Ruggieri d'Aieroli, uomo "di nazione nobile ma di cattiva vita”, ovvero l'esatto contrario di Guiscardo ${ }^{3}$.

Dunque, per un verso, tutte e due le giovani decidono le proprie azioni sottoponendole al pieno controllo razionale; lontane da qualunque impulsività e cedimento all'istinto, come anche da ribellioni o rivalse aperte, considerano, meditano, valutano gli elementi della loro situazione e le modalità di iniziative che possano soddisfare quanto viene a loro indebitamente negato. Per l'altro, da questo punto in poi loro strade si divaricano. Ghismonda pagherà con la vita questo sapiente e virtuoso esercizio, avendo governato da sola e con piena responsabilità morale lo svolgimento degli eventi e le motivazioni delle azioni del padre/tiranno, fino al fatale esito, rivendicato come opzione liberamente assunta. In questo mostrando (come altre protagoniste decameroniane) la superiorità di chi sappia, in qualunque circostanza, anche estrema, addomesticare la fortuna mediante l'utilizzo della ragione, dell'ingegno, insomma dell'intelligenza.

Si tratta di uno dei segnali più significativi dell'ispirazione latamente stoica che anima molti personaggi decameroniani, pronti consapevolmente a sacrificare anche la vita per affermare fino in fondo e a ogni costo la volontà di controllo su ogni fase della propria esistenza e quindi anche della propria morte 4 . Il suicidio perciò, in questa prospettiva, non è un atto di rinuncia, ma al contrario di affermazione di sé e della propria libertà. Così lo intenderà molti secoli dopo Leopardi che su questa idea svilupperà pagine dello Zibaldone e composizioni poetiche come Bruto minore e Ultimo canto di Saffo, tra le altre. Naturalmente la distanza tra Boccaccio e Leopardi è grande per moltissimi aspetti; tuttavia ritrovarli, per questo aspetto, sulla medesima linea di pensiero non è un dato marginale, tantomeno casuale perché richiama l'enorme suggestione che lo stoicismo ha esercitato per secoli sulla cultura europea e italiana, in particolare nella versione che ne ha dato Seneca nelle opere filosofiche e in quelle drammaturgiche.

All'ingegno congiunto alla virtù che anima l'agire di Ghismonda corrisponde la condizione di soggezione al dominio della fortuna (boccaccianamente intesa) che costringe la moglie del medico a ricorrere all'aiuto dell' astuta fante, la vera artefice del lieto scioglimento dell'intrigo. La catena degli avvenimenti è avviata, in entrambi i casi, da fortuiti accidenti (Tancredi addormentato nella camera della figlia che assiste, non visto, agli amori consumati con Guiscardo e, nell'altra novella, la sete che induce Ruggieri a bere il sonnifero, dando il via così all'intrigo

3 Com'è opportunamente notato da Branca (BOCCACCIO, 1987, p. 572), che tuttavia, pur sottolineando i frequenti rovesciamenti comici che costellano la novella, non sembra coglierne la natura strutturante.

4 Per le suggestioni senecane nel Decameron cfr. G. Velli, Memoria, in Lessico critico decameroniano, a cura di R. Bragantini e P.M. Forni, Bollati Boringhieri, Torino 1995, p. 222-248; G. Velli, Seneca nel “Decameron”, in Petrarca e Boccaccio. Tradizione-memoria-scrittiura, Antenore, Padova 1991, p. 209-221). 
farsesco) ma si snoda lungo due percorsi esattamente opposti: tragico in ogni passaggio e funesto il primo, comico e buffonesco il secondo, con una puntuale corrispondenza all'incontrario.

E però in entrambi i casi, pure così diversi e distanti, la ragione, l'ingegno delle donne (pure variamente impegnato) prevalgono sull'emotività maschile, congiunta questa all'esercizio (anche brutale, nel caso di Tancredi) del potere paterno e maritale; questo dato, in ultima analisi, consente alle due protagoniste di dominare la storia in tutto il suo svolgimento, fino all'esito che sancisce per entrambe il riconoscimento anche sociale del loro successo.

Così doloroso fine ebbe l'amor di Guiscardo e di Ghismonda, come udito avete: li quali Tancredi dopo molto pianto e tardi pentuto della sua crudeltà, con general dolore di tutti i salernetani, onorevolmente ammenduni in un medesimo sepolcro gli fè sepellire. (IV, 1, 62).

Alla fine [lo stradicò], cognoscendo Ruggieri essere innocente, condannati li prestatori che imbolsta avevan l'arca in diece once, liberò Ruggieri; il che quanto a lui fosse caro, niun ne domandi, e alla sua donna fu carissimo oltre misura. La qual poi con lui insieme e con la cara fante, che dare gli aveva voluto delle coltella, più volte rise e ebbe festa, il loro amore e il loro sollazzo sempre continuando di bene in meglio: il che vorrei che così a me avvenisse ma non d'esser messo nell'arca. (IV, X, 53).

Il lieto e giocoso finale della IV, 10 si presenta con tutta evidenza come l'esatto opposto del tragico esito della IV, 1. Particolarmente le ultime parole del narratore richiamano, a rovescio ancora una volta, quelle pronunciate dal re della IV giornata, Filostrato, all'inizio della novella IV , 2, come commento alla precedente, un commento del tutto inusuale perché decisamente personalizzato 5 :

Poco prezzo mi parrebbe la vita mia a dover dare per la metà diletto di quello che con Guiscardo ebbe Ghismonda, né se ne dee di voi maravigliare alcuna, con ciò sia cosa che io, vivendo, ogni ora mille morti sento, né per tutte quelle una sola particella di diletto m'è data. (IV, 2, 2).

5 Mi sono soffermata su questo aspetto nel saggio Tragico e comico in due novelle del Decameron (STRAPPINI, 2010), già citato. 
Si può cogliere un ulteriore elemento di confronto nella funzione operativa svolta dalla bevanda, mortale per Ghismonda che berrà volontariamente il veleno versato nella coppa d'oro contenente il cuore di Guiscardo (fatto strappare da suo padre all'amante assassinato) e dalla bevanda con il sonnifero preparata dal medico per un suo paziente e inavvertitamente bevuta dal Ruggieri, fatto che dà origine a tutto l'intrigo che lo condurrebbe alla condanna e alla morte se non venisse salvato dalla sagacia della donna. Il motivo drammatico del cuore mangiato, ripreso da Boccaccio da una riconoscibile tradizione medievale6, è ulteriormente nobilitato dalla magnanima determinazione di Ghismonda:

E questo detto, si fe' dare l'orcioletto nel quale era l'acqua che il dì davanti aveva fatta, la quale mise nella coppa ove il cuore era da molte delle sue lagrime lavato; e senza alcuna paura postavi la bocca, tutta la bevve e bevutala con la coppa in mano se ne salì sopra il suo letto, e quanto più onestamente seppe compose il corpo suo sopra quello e al suo cuore accostò quello del morto amante; e senza dire alcuna cosa aspettava la morte.

Rimane, nella IV, 10, l'acqua alterata (qui, appunto, non con il veleno, ma con il sonnifero), però la severa risolutezza diviene infortunio accidentale e alla gravità di Ghismonda distesa "onestamente" sul letto in attesa silenziosa della morte, corrisponde la caduta a terra del giovane addormentato dal sonnifero, come "un corpo morto"

Standosi adunque Ruggieri nella camera e aspettando la donna , avendo o per fatica il dì' durata o per cibo salato che mangiato avesse o forse per usanza una grandissima sete, gli velle nella finestra veduta questa guastadetta d'acqua la quale il medico per lo 'nfermo aveva fatta, e credendola acqua da bere, a bocca postalasi, tutta la bevé; né stette guari che un gran sonno il prese, e fusi adormentato. (IV, $10,13)$.

e lo spavento della moglie del medico, lo smarrimento e il ricorso a una via d'uscita di fortuna

6 Cfr. Mariella Di Maio, Il cuore mangiato. Storia di un tema letterario dal Medioevo all'Ottocento, Guerini e associati, Milano 1996. 
ma dopo alquanto, temendo la donna di non aggiungere al suo danno vergogna, pensò che senza alcuno indugio da trovare era modo come lui morto si traesse di casa; né a ciò sappiendosi consigliare, tacitamente chiamò la sua fante e la sua disavventura mostratale le chiese consiglio. (IV, 10, 17).

Per cogliere puntualmente tutto il gioco incrociato sarebbe necessario leggere in parallelo le due novelle nella loro integralità. Potrà essere sufficiente qui avere indicato alcuni tratti salienti in quella direzione che consentono di verificare come il percorso narrativo delle due novelle sia modulato su sequenze analoghe e soprattutto che è il rovesciamento del tragico della prima nel comico della seconda a costituire il senso essenziale del nesso tra le due narrazioni e le loro conclusioni, a conferma di quella modalità di rappresentazione e di narrazione tipicamente boccacciana che è fondata sulla congiunzione/complementarietà di tragico e di comico in quanto tratto massimamente significante della vicenda umana sotto tutti i profili ${ }^{7}$.

Certo, anche nel grande modello dantesco della Commedia i due toni sono egualmente presenti e attivi. Nel Decameron questa visione è posta precisamente al servizio del ritratto del mondo terreno, tutto il mondo terreno, nelle sue svariate manifestazioni. Non solo, perciò, come nella Commedia, la scrittura si modula sulla tonalità del tragico accanto, a volte, o insieme alla tonalità del comico, in modo che entrambe siano al massimo valorizzate; ma, in più, si diletta ${ }^{8}$ a proporre la versione comica della vicenda tragica (e viceversa) con l'ambizione, peraltro ben riuscita, di riprodurre nella narrazione, nella letteratura, la sconfinata varietà delle esistenze umane.

7 Rimando anche su questo punto all'articolo già citato, Tragico e comico in due novelle del Decameron (STRAPPINI, 2010).

8 Sono molte le analisi e le riflessioni degli studiosi sul diletto del narrare come perno centrale dell'ispirazione boccacciana e della sua idea della civiltà e dell'efficacia del vivere. Viene considerata esemplare, in questo senso, la novella IV, 1 (Madonna Oretta), ma in effetti questo filo attraversa proficuamente l'intera opera che potrebbe essere riletta sotto questo prevalente profilo. 


\section{Riferimenti bibliografici}

BOCCACCIO, G. Decameron. A cura di Vittore Branca. Torino: Einaudi, 1987.

DI MAIO, M. Il cuore mangiato. Storia di un tema letterario dal Medioevo all'Ottocento. Milano: Guerini, 1996.

STRAPPINI, L. Tragico e comico in due novelle del Decameron. In Revista de Italianística, n. XIXXX, 2010, p. 17-29.VELLI, G. Memoria. In BRAGANTINI, R.; FORNI, P. M. (a cura di). Lessico critico decameroniano. Torino: Bollati Boringhieri, 1995. p. 222-248.

VELLI, G. Seneca nel Decameron. In . Petrarca e Boccaccio. Tradizione, memoria, scrittura.

Padova: Antenore, 1991. p. 209-221. 